\title{
Preoperative Assessment Using Magnetic Resonance Imaging for Subungual Exostosis beneath the Proximal Region of the Nail Plate
}

\author{
Kuriko Higuchi Naoki Oiso Masuki Yoshida Akira Kawada \\ Department of Dermatology, Kinki University Faculty of Medicine, Osaka-Sayama, Japan
}

\section{Key Words}

Preoperative assessment · Magnetic resonance imaging · Subungual exostosis .

Nail plate $\cdot$ Nail matrix $\cdot$ Onycholysis

\begin{abstract}
Subungual exostosis is a benign calcifying projection that occurs on the distal phalanx beneath or beside the nail. The problems in treatment are frequent postoperative recurrence and nail deformity or loss. Here, we describe the case of a male patient with a subungual exostosis beneath the proximal region of the nail plate of the right big toe. A precise preoperative evaluation of the anatomical structure using magnetic resonance imaging was useful for removing the subungual exostosis.
\end{abstract}

\section{Introduction}

Subungual exostosis is a benign calcifying projection that occurs on the distal phalanx beneath or beside the nail. Radiography reveals an outgrowth of the trabecular bone. The problems in treatment are frequent postoperative recurrence because of incomplete removal, and nail deformity or loss due damage to the nail matrix [1]. Here, we present the case of a 21-year-old man with a subungual exostosis beneath the proximal region of the nail plate of the right big toe. It was successfully treated by means of preoperative assessment using magnetic resonance imaging (MRI).

\section{Case Report}

A 21-year-old man was referred to our department with a slow-growing tumor on the right big toe. He had noticed it 6 months earlier. Physical examination revealed a pale whitish nodule with tenderness, $7 \mathrm{~mm}$ in diameter, beneath the proximal region of the nail plate (arrow in fig. $1 \mathrm{a}$ ). 


\begin{abstract}
Onycholysis was present toward the distal region (fig. 1a). A radiograph indicated a calcifying projection on the distal phalanx continuous with the underlying bone (arrow in fig. 1b). Sagittal T1weighted MRI showed that the projection was high signal intensity (arrow in fig. 1c) encircled by low signal intensity (the fibrous cartilage) (fine arrow in fig. 1c), and that the connective tissue including the nail matrix was intermediate signal intensity (dotted arrow in fig. 1c). The proximal region of the tumor lies under the nail matrix (fig. 1c). After removal of the nail plate, a longitudinal fish-mouth-type incision was made to lift the entire nail bed and expose the tumor which was then removed as completely as possible [2]. HE staining showed a base of lamellated trabecular bone covered by fibrous cartilage (fig. 1d). The preservation of the nail matrix and sufficient removal of subungual exostosis allowed for complete recovery of the nail plate without recurrence for 1 year.
\end{abstract}

\title{
Discussion
}

Surgical treatment for subungual exostosis requires removal of a margin of normal bone to prevent recurrence, and preservation of the nail matrix to avoid nail deformity [ 1 , 2]. A radiograph can identify a calcifying projection. The image diagnosed the subungual tumor with subungual exostosis. However, it cannot supply the anatomical image between the calcifying projection and the nail matrix. Sagittal T1-weighted MRI can show a more precise illustration between calcifying projection and connective tissue of the nail matrix than computed tomography or ultrasonography. Therefore, we used MRI to confirm whether the tumor beneath the proximal region of the nail plate undermined the nail matrix. Preoperative confirmation of the attachment of the tumor to the nail matrix prompted us to design and perform the removal cautiously.

The innovation of MRI allows for more specific analysis of the nail apparatus [3, 4]. MRI may be useful for preoperative assessment of disorders involving the nail apparatus. 

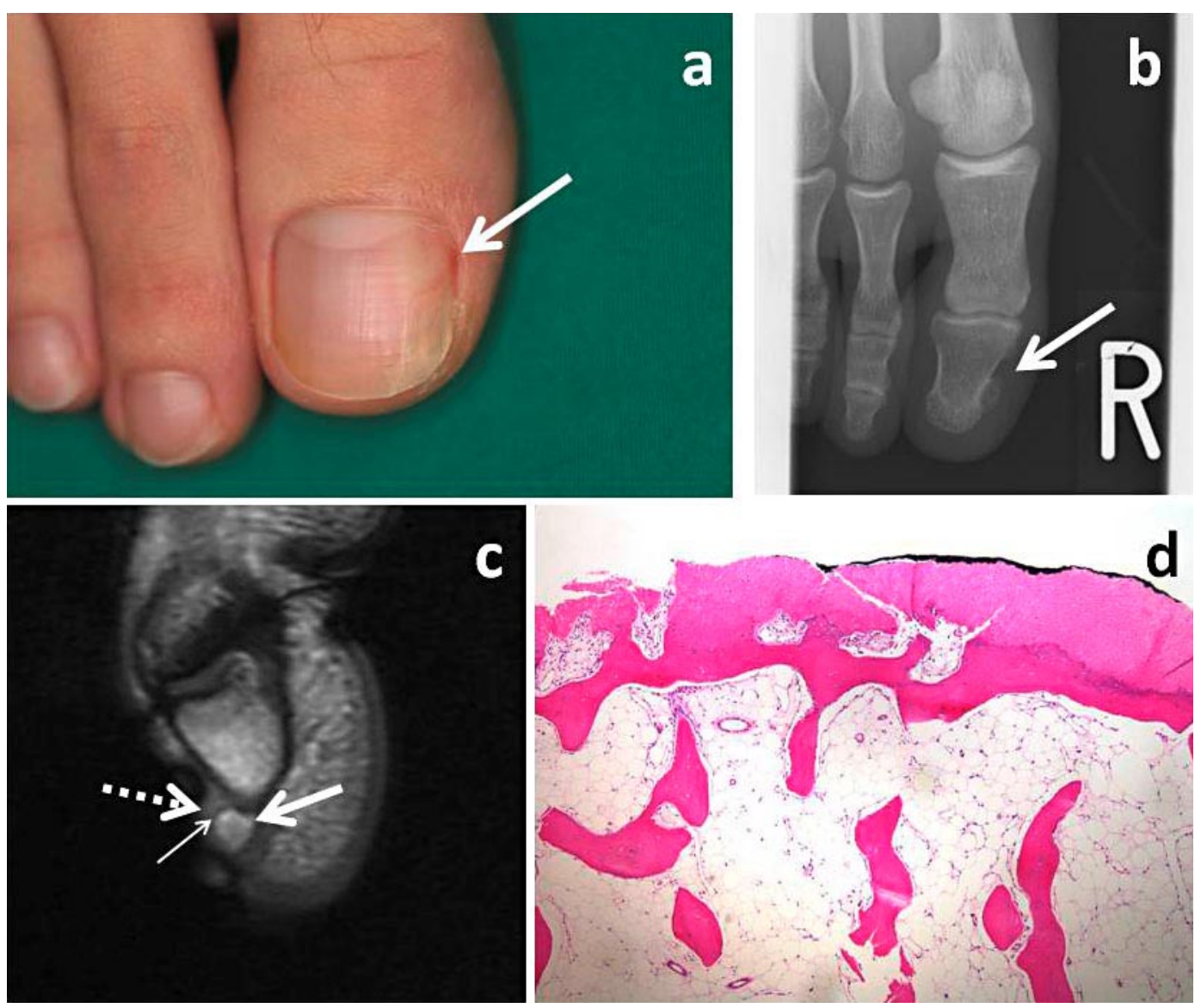

Fig. 1. a Clinical image of the subungual exostosis (arrow) complicated by onycholysis. b Radiograph of the subungual exostosis (arrow). c Sagittal T1-weighted MRI of the subungual exostosis showing the projection as high signal intensity (arrow), the fibrocartilaginous cap as low signal intensity (fine arrow), and the connective tissue including the nail matrix as intermediate signal intensity (dotted arrow). d Histopathological image of the subungual exostosis, with lamellated trabecular bone covered by fibrous cartilage: HE staining, original magnification $\times 40$.

\section{References}

1 de Berker DA, Baran R: Disorders of nails. Subungual exostosis; in Burns T, Breathnach S, Cox N, Griffiths C (eds): Rook's Textbook of Dermatology. 8th ed. Chichester: A John Wiley and Sons, Ltd. Publication, 2010, vol 4, pp 65.33-65.34.

2 Dumontier CA, Abimelec P: Nail unit enchondromas and osteochondromas: a surgical approach. Dermatol Surg 2001;27:274-279.

-3 Baek HJ, Lee SJ, Cho KH, Choo HJ, Lee SM, Lee YH, Suh KJ, Moon TY, Cha JG, Yi JH, Kim MH, Jung SJ, Choi JH: Subungual tumors: clinicopathologic correlation with US and MR imaging findings. Radiographics 2010;30:1621-1636.

4 Richert B, Baghaie M: Medical imaging and MRI in nail disorders: report of 119 cases and review of the literature. Dermatol Ther 2002;15:159-164. 